Review

\title{
The Role of p38y in Cancer: From review to outlook
}

\author{
Wentao $\mathrm{Xu}^{1,2^{*}}$, Rui $\mathrm{Liu}^{1 *}$, Ying Dai ${ }^{1}$, Shaocheng Hong ${ }^{2}$, Huke Dong2 ${ }^{2}$, Hua Wang1, ${ }^{\circledR}$ \\ 1. Department of Oncology, the First Affiliated Hospital of Anhui Medical University, Hefei, 230022, Anhui, China. \\ 2. First Clinical Medical College of Anhui Medical University, Hefei, 230032, Anhui, China. \\ 3. Inflammation and Immune Mediated Diseases Laboratory of Anhui Province, Anhui Medical University, Hefei, 230032, Anhui, China. \\ *These authors contributed equally to this work. \\ $\square$ Corresponding author: Hua Wang, M.D., Ph.D., Department of Oncology, the First Affiliated Hospital of Anhui Medical University; 218 Jixi Road, Hefei, \\ 230022, Anhui, China. E-mail: wanghua@ahmu.edu.cn. \\ ( ) The author(s). This is an open access article distributed under the terms of the Creative Commons Attribution License (https://creativecommons.org/licenses/by/4.0/). \\ See http://ivyspring.com/terms for full terms and conditions.
}

Received: 2021.06.04; Accepted: 2021.09.16; Published: 2021.09.23

\begin{abstract}
$p 38 y$ is a member of the $\mathrm{p} 38$ Mitogen Activated Protein Kinases (p38 MAPKs). It contains four subtypes in mammalian cells encoded by different genes including $p 38 \alpha$ (MAPK14), p38 $\beta$ (MAPK11), $p 38 \gamma$ (MAPK12), and $p 38 \delta$ (MAPK13). Recent studies revealed that $p 38 \gamma$ may exhibit a crucial role in tumorigenesis and cancer aggressiveness. Despite the large number of published literatures, further researches are demanded to clarify its role in cancer development, the tissue-specific function and associated novel treatment strategies. In this article, we provide the latest view on the connection between $\mathrm{p} 38 \mathrm{y}$ and malignant tumors, highlighting the function of $\mathrm{p} 38 \mathrm{\gamma}$. The clinical value of $\mathrm{p} 38 \mathrm{\gamma}$ is also discussed, helping the translation into the remarkable therapeutic strategy in tumor diseases.
\end{abstract}

Key words: p38y, molecular pathway, intestinal microbes, gene editing, immunotherapy

\section{Introduction}

To date, the p38y, the third mammalian p38, is initially described as a novel protein kinase from rats [1], known to assist local phosphorylation in the cell nucleus [2]. p38 $\mathrm{\gamma}$ is a $43-\mathrm{kDa}[3]$ and 367 -amino acid protein [4] with a characteristic C-terminal motif KETXL. It can be docked with the PSD-95/Dlg/ZO-1 homology (PDZ) domain of additional proteins, such as a 1-syntrophin, PTPN3, SAP90/PSD95 and the scaffold protein SAP97/hDlg. p38 $\gamma$ binds its PDZ domain of these proteins and induces the phosphorylation $[5,6]$. For instance, the environmental osmotic pressure changes trigger $\mathrm{p} 38 \gamma$ activation in the cytoplasm and concentrate in the nucleus, thereby increasing the binding of $p 38 \mathrm{\gamma}$ and nuclear $\mathrm{hDlg}$ and resultantly disassembly of the hDlg-PSF (protein-associated-splicing factor) complex $[7,8]$. Due to its capacity to shuttle between cytoplasm and nucleus, $\mathrm{p} 38 \mathrm{\gamma}$ might establish a link between two key procedures of gene expression and cytoskeleton reorganization to adapt to environmental changes.

Notably, p38y can be activated by the stress and mitogenic signals, and further promote tumorigenesis and progression of cancer [9]. For example, $\mathrm{p} 38 \gamma$ is activated by Ras and is indispensable for Ras transformation and invasion $[2,10,11]$. p38 $\gamma$ is located predominantly in the nucleus [3]. Meanwhile p38 $\mathrm{y}$ is capable of inhibiting cell migration, essential for oncogene-induced senescence [12]. These findings indicate that $\mathrm{p} 38 \mathrm{\gamma}$ probably has tumor suppressive or carcinogenic effects (Table 1 ).

\section{Overview of MAPKs}

Mitogen-activated protein kinases (MAPKs) are serine/threonine protein kinases that convert extracellular stimuli into a variety of cellular responses [13]. The RAS/RAF/MEK/ERK (MAPK) signaling cascade, one of the widespread signaling pathways, plays a vital role in cellular processes whose cell disorders relevant to carcinogenesis and cancer development, including differentiation, propagation, inflammation, apoptosis, survival and innate immunity [13]. In the early 80s, ERK1 appeared in the focus as the first MAPK core molecule to be discovered and identified in mammals [14]. Subsequently, the highly homologous ERK2 and ERK3 were cloned one after another, and the research 
on the mammalian MAPK pathway began [14]. Currently, typical MAPKs mainly include ERK1/2, JNK1/2/3, p38 isoforms accompanied by in-depth studies [14]. Furthermore, each classic MAPK consists of a set of three kinases acting in sequence: a MAPK, a MAPK kinase (MAPKK) and a MAPKK kinase (MAPKKK), and the extensive functions they regulate are principally mediated by phosphorylation of members of the protein kinase family named MAPK-activated protein kinases (MAPKAPKs) [14].

\section{Overview of p38}

The p38 MAPK family is one of the four major mitogen-activated protein kinases (MAPKs) cascades as a class of highly conserved proteins found firstly in studies of endotoxin-induced cytokine expression [10, 15-17]. It was reported to get involved in inflammation, cell growth, cell differentiation, cell death, and the cell cycle [10, 15, 18-21]. So far, there are four p38 isoforms respectively named p38a (MAPK14), p38 $\beta$ (MAPK11), p38y (SAPK3, ERK6 or MAPK12), and p38ס (MAPK13) [5]. These kinases share extremely analogical protein sequences; $\mathrm{p} 38 \mathrm{a}$ and $\mathrm{p} 38 \beta$ have $75 \%$ identity, whereas p38 $\gamma$ and $\mathrm{p} 38 \delta$ have $62 \%$ and $61 \%$ identity with p38a, respectively. Simultaneously, p38 $\gamma$ and $\mathrm{p} 38 \delta$ have $70 \%$ similarity with each other [22].

\section{p38y and Cell cycle}

Targetedly, cells are capable to dispose DNA damage by terminating the cell cycle process or performing procedural cell death [23]. Highly conservative DNA repair and cell cycle checkpoint pathways enable cells to cope with endogenous and exogenous DNA damage [23].

In the past decade, p38 pathway has been emphasized as a core part of cell cycle regulator in cancer which principally manifested as the effect of $\mathrm{p} 38 \mathrm{a} / \beta / \gamma$ instead of p388 (Figure 1). For example, in human breast cancer MDA-MB-231BO cells, hyaluronan activates $p 38 \mathrm{a} / \beta$ and up-regulates the expression of $\mathrm{p} 21^{\mathrm{cip} 1}$, leading to the decrease of Cyclin D1 level, prolonging the conduction in G0-G1 phase and slowing the growth of breast cancer cells [24]. p38a can also phosphorylate $\mathrm{Rb}$, down-regulate the expression of E2F transcription factor, delay the progression of G1-S phase and reduce tumor cell proliferation in MCF7 breast cancer cells [25]. Likewise, the activation of p38a-dependent p53 promotes the expression of GADD45a, inhibits the activity of the Cdc2/Cyclin B1 complex, and achieves the function of inhibiting the growth of HCT116 human colorectal carcinoma cells by blocking the G2-M phase [26]. A study has reported that circTNFRSF21 is the upstream of p38ס, and its expression enhancement can promote the transition of endometrial carcinoma cells from G0/G1 to S phase. However, the mechanism by which $\mathrm{p} 38 \delta$ regulates the cell cycle is still unclear [27].

Table 1. The carcinogenic and anticancer effects of $p 38 y$

\begin{tabular}{|c|c|c|c|}
\hline Type & Observation & Species & References \\
\hline \multicolumn{4}{|c|}{ Tumors promoted by p38 $\gamma$ signaling } \\
\hline \multirow[t]{4}{*}{$\mathrm{BC}$} & $\begin{array}{l}\mathrm{p} 38 \gamma \text { is related to the aggressiveness and metastasis } \\
\text { of } B C\end{array}$ & Human & $\begin{array}{l}11,40,41 \\
42,43,45\end{array}$ \\
\hline & $\begin{array}{l}\text { Alcohol selectively stimulates phosphorylation of } \\
\text { p38y in breast cancer cells }\end{array}$ & Mouse & 43 \\
\hline & $\begin{array}{l}\text { Increased } \mathrm{p} 38 \mathrm{y} \text { expression is related to a decrease in } \\
\text { overall survival rate of } \mathrm{BC} \text { patients }\end{array}$ & Human & 41 \\
\hline & $\begin{array}{l}\text { PFD acts as a targeted inhibitor of p38 } \mathrm{y} \text { and can } \\
\text { inhibit the development of TNBC in cells and/or } \\
\text { mice }\end{array}$ & Mouse & 48 \\
\hline \multirow[t]{3}{*}{ SCC } & $\begin{array}{l}\text { SCC incidence and tumor lesion number per mouse } \\
\text { were lower in p38 } \gamma \text { - mice than that in WT mice }\end{array}$ & Mouse & 21 \\
\hline & $\begin{array}{l}\text { There is a conspicuous correlation between the } \\
\text { expression of p38y and the clinical stage of tumor, } \\
\text { tumor volume and lymph node metastasis in ESSC }\end{array}$ & Human & 49 \\
\hline & The expression extent of $\mathrm{p} 38 \gamma$ is related to HNSCC & Human & 20 \\
\hline \multirow[t]{3}{*}{$\mathrm{HCC}$} & $\begin{array}{l}\text { In HCC cases, the excessive expression of } \mathrm{p} 38 \mathrm{\gamma} \text { is } \\
\text { highly indicative of its carcinogenicity and } \\
\text { unfavorable prognosis }\end{array}$ & Human & 31 \\
\hline & $\begin{array}{l}\text { In mice with absent } \mathrm{p} 38 \mathrm{\gamma}, \mathrm{HCC} \text { is strongly inhibited, } \\
\text { and survival time is prolonged }\end{array}$ & Mouse & 31 \\
\hline & $\begin{array}{l}\text { p38y inhibitor PFD is able to prevent the occurrence } \\
\text { of HCC induced by DEN }\end{array}$ & Mouse & 31 \\
\hline \multirow[t]{2}{*}{ CRC } & $\begin{array}{l}\text { Highly expressed } \mathrm{p} 38 \mathrm{\gamma} \text { boosts the growth, } \\
\text { proliferation, cell migration and apoptosis resistance } \\
\text { of CRC cells in human CRC tissues }\end{array}$ & Human & 34 \\
\hline & $\begin{array}{l}\text { Mice with IEC-specific } \mathrm{p} 38 \gamma \text { deletion in the } \\
\text { AOM/DSS model have reduced colon tumor } \\
\text { formation }\end{array}$ & Mouse & 51 \\
\hline \multirow[t]{2}{*}{ PDAC } & $\begin{array}{l}\text { p38 } \gamma \text { is involved in the spread of PDAC and } \\
\text { aggressive behavior }\end{array}$ & Mouse & 16 \\
\hline & $\begin{array}{l}\text { The pharmacological p38 } \gamma \text { inhibitor PFD inhibits } \\
\text { carcinogenesis effect and tumor growth in PDAC }\end{array}$ & Mouse & 16 \\
\hline Glioma & $\begin{array}{l}\mathrm{p} 38 \gamma \text { is related to the proliferation and apoptosis of } \\
\text { glioma, and } \mathrm{p} 38 \mathrm{\gamma} \text { is positively correlated to the } \\
\text { differentiation grade of glioma }\end{array}$ & Human & 55 \\
\hline CTCL & $\begin{array}{l}\text { High level of p38 } \gamma \text { is related to CTCL and is } \\
\text { fundamental for viability of cancer }\end{array}$ & Human & 4,57 \\
\hline OS & $\begin{array}{l}\mathrm{p} 38 \mathrm{y} \text { may play an indispensable part in the } \\
\text { proliferation, migration and invasion of OS cells }\end{array}$ & Human & 58,36 \\
\hline \multirow[t]{3}{*}{ RCC } & $\begin{array}{l}\text { Overexpression of } \mathrm{p} 38 \mathrm{\gamma} \text { is capable to encourage RCC } \\
\text { cell development, proliferation and migration }\end{array}$ & Human & 35,60 \\
\hline & $\begin{array}{l}\text { The expression of } \mathrm{p} 38 \mathrm{\gamma} \text { mRNA and protein in RCC } \\
\text { tissues are higher than those in normal tissues } \\
\text { adjacent to the carcinoma }\end{array}$ & Human & 35 \\
\hline & $\begin{array}{l}\text { High p38y levels are detected in FeNTA-induced } \\
\text { renal cancer development and maintenance }\end{array}$ & Human & 60 \\
\hline NSCLC & $\begin{array}{l}\text { Compared with the control group, the markedly } \\
\text { elevated level of p38y is related to the germination of } \\
\text { NSCLC disease }\end{array}$ & Human & 61 \\
\hline $\begin{array}{l}\text { Bladder } \\
\text { cancer }\end{array}$ & $\begin{array}{l}\text { The outcome of patients with high expression of } \mathrm{p} 38 \mathrm{y} \\
\text { is worse }\end{array}$ & Human & 62 \\
\hline \multicolumn{4}{|c|}{ Tumors suppressed by p38 $\gamma$ signaling } \\
\hline NSCLC & $\begin{array}{l}\text { In xenograft models, } \mathrm{p} 38 \mathrm{\gamma} \text { is able to lessen the tumor } \\
\text { initiation ability and tumor growth of NSCLC cells }\end{array}$ & Mouse & 12 \\
\hline
\end{tabular}

Abbreviations: BC, breast cancer; TNBC, triple-negative breast cancer; SCC, squamous cell cancer; HNSCC, head and neck squamous cell carcinoma; ESSC, esophageal squamous cell carcinoma; CRC, colorectal cancer; HCC, hepatocellular carcinoma; PDAC, pancreatic ductal adenocarcinoma; RCC, renal cell carcinoma; CTCL, cutaneous t-cell lymphoma; OS, osteosarcoma; NSCLC, non-small cell lung cancer; GBM, glioblastoma; WT, wild type; IEC, intestinal epithelial cell; PFD pirfenidone; AOM/DSS, azomethane/dextran sodium sulfate; DEN, diethylnitrosamine. 

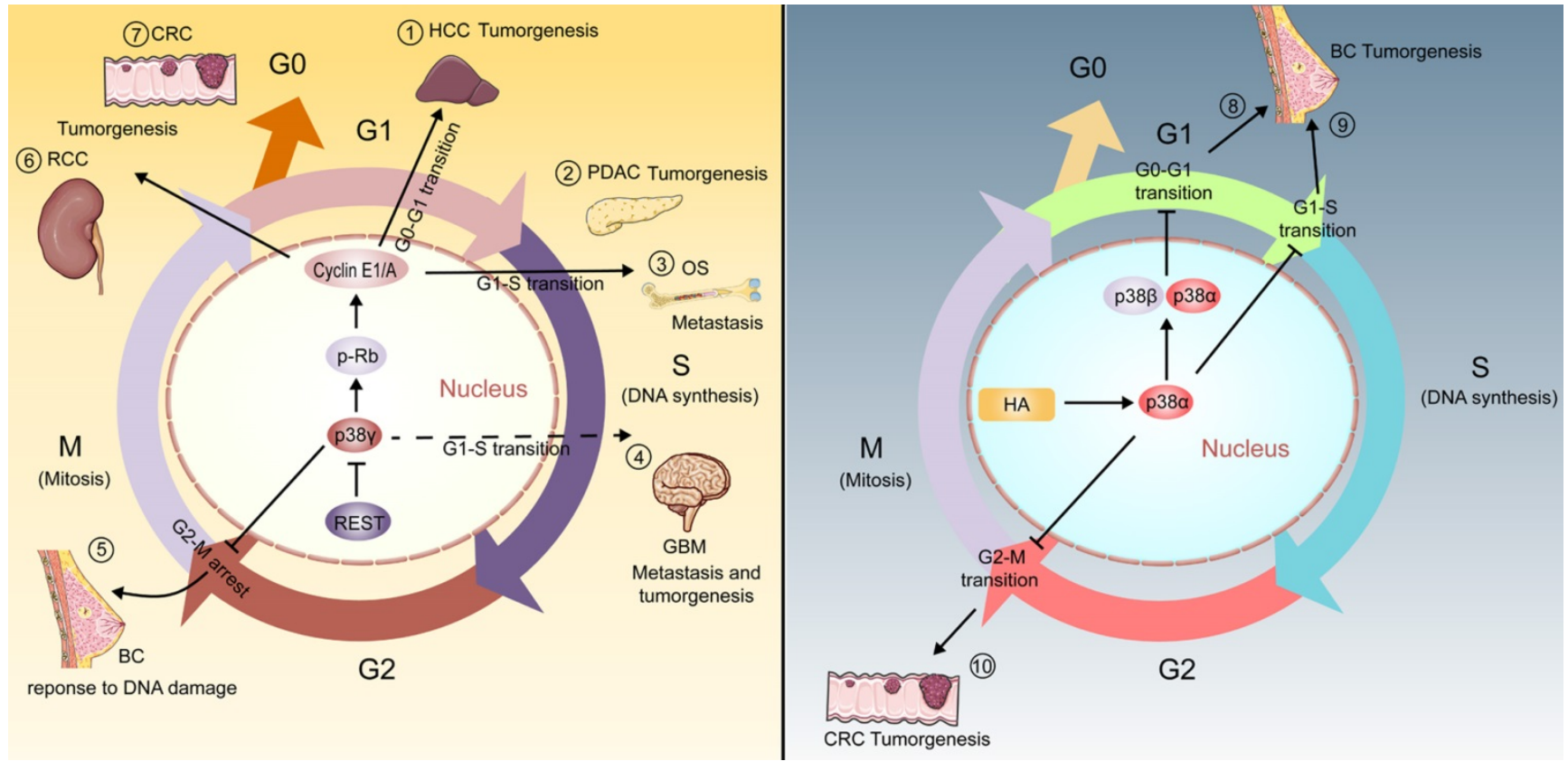

Figure 1. p38y participates in the biological process of various cancers by regulating the cell cycle process. (1) p38y/p-Rb/Cyclin (E1/A) pathway could encourage tumorigenesis and proliferation of hepatocellular carcinoma by promoting G0-G1 phase transition; (2) p38y/p-Rb/Cyclin (E1/A) pathway could promote the transition of the cell cycle from G1 to S phase, and is affiliated to tumorigenesis, proliferation, and motility of pancreatic ductal carcinoma; (3) p38y/p-Rb/Cyclin (E1/A) pathway could promote the transition of the cell cycle from G1 to S phase, connected with tumorigenesis, proliferation, and invasion of osteosarcoma; (4) As a downstream target of transcription factor REST, p38y can be restrained by REST and may promote the transition of G1-S phase, resulting in the proliferation and migration of glioblastoma; (5) P38y causes G2-M phase arrest and maintains breast cancer cell survival under conditions of DNA damage; (6) p38y/p-Rb/Cyclin (E1/A) pathway may play a crucial part in the proliferation, development and migration of human renal cell carcinoma cells; (7) p38y/p-Rb/Cyclin (E1/A) pathway may play a vital role in the proliferation, development and invasion of human colorectal cancer cells; (8) Hyaluronan-dependent $\mathrm{P} 38 \alpha / \beta$ activation induces GO-G1 phase arrest, leading to growth inhibition of breast cancer; (9) Blocking G1-S phase conduction by p38 $\alpha$ also inhibits the tumorigenesis of breast cancer; (10) p38 $\alpha$ blocks G2-M phase transmission and weakens the tumorigenesis of colorectal cancer.

As early as 1999, p38y has been found to inhibit cell proliferation by down-regulating cyclin D1 during adrenal cell hypoxia [28]. Moreover, it is proved that the activation of ATM/MKK6/p38y/ Cds1 pathway is essential for the correct regulation of G2 checkpoints in mammalian cells [29]. In line with this, it has been proved that Xenopus $\mathrm{p} 38 \mathrm{\gamma}$ can promote the meiosis of Xenopus oocytes treated with progesterone, while p38a or p38 $\beta$ have no effect [30]. On this basis, fully grown Xenopus oocytes are blocked in prophase of meiosis I and are induced to pass through meiosis by progesterone stimulation [30]. In human cells, it has also been found that the presence of $\mathrm{p} 38 \mathrm{\gamma}$ can cause cell cycle changes, and this may be essential for the proliferation, invasion and carcinogenic activity of numerous cancers [16, 31]. Similarly, the ectopic expression of p38y may contribute to a significant arrest of the cell cycle in G2/M phase, which may be a mechanism of breast cancer cells to survive established on DNA damage $[32,33]$.

Taken together, $\mathrm{p} 38 \mathrm{\gamma}$ participates in the regulation of the cell cycle, contributing to a variety of cancers [16, 31, 32, 34-37] (Figure 1). Considering the prominent position of $\mathrm{p} 38 \mathrm{\gamma}$ in regulating the cell cycle process, the role of $\mathrm{p} 38 \mathrm{y}$ in cell cycle regulation in more cancers is worthy of further investigation.

\section{Breast Cancer}

Estrogen receptor (ER) is a transcription factor related to $\mathrm{BC}$, which has the paradoxical effect of promoting proliferation and resisting invasion $[11,38$, 39]. It has been reported that $\mathrm{p} 38 \mathrm{\gamma}$ can integrate the invasive antagonism of Ras and ER without affecting the proliferative activity of $\mathrm{BC}$, thereby promoting the invasiveness of BC [11]. Involvement in cellular metabolism is another mechanism by which $\mathrm{p} 38 \mathrm{\gamma}$ increases BC invasiveness. Using a novel metabolomics approach to demonstrate the role of p38 $\gamma$ in apoptosis, invasion, and migration, Chen et al. showed the potential to promote cancer metastasis, particularly altering the enrichment of certain metabolites and metabolic pathways, which may bring potential to the evolution of novel therapeutic strategies for BC [40].

Another study based on a particular kinetic cell movement analysis method further certifies that $\mathrm{p} 38 \mathrm{\gamma}$ can control cell movement by regulating the metastasis-associated small GTPase-RhoC ubiquitination and lysosomal degradation, as well as regulate diversifications in cell structure and cell form of BC [41]. This evidence suggests p38 $\gamma$ may possess a fundamental effect in the metastatic properties of BC. Apart from that, there is pertinence between 
enhancive expression of $\mathrm{p} 38 \mathrm{\gamma}$ and the decline in the overall survival rate of $\mathrm{BC}$ patients [41].

In ethanol-induced mice, loss of $\mathrm{p} 38 \mathrm{\gamma}$ can alleviate BC development and metastasis, and knockdown of p38 $\gamma$ can block alcohol-aroused RhoC activation, cell spread, invasion and migration [42]. Correspondingly, there is a significant positive correlation between the expression of $\mathrm{p} 38 \mathrm{y}$ and RhoC in clinical BC samples [41]. Alcohol has also been reported to augment the invasiveness of $\mathrm{BC}$ cells in another study. It stimulates the phosphorylation of p38y by activating ErbB2 and promotes the interaction of $\mathrm{p} 38 \gamma$ with the substrate SAP97/DLG as well as the phosphorylation of SAP97/DLG, thus leading to the activation of CSCs (cancer stem cells) [43].

Epithelial cell-mesenchymal transition (EMT) refers to the biological process of epithelial cells transforming into cells with mesenchymal phenotype through specific procedures and helps cancer cells spread from solid tumors and form detectable metastasis [44]. It was previously shown that $\mathrm{p} 38 \mathrm{\gamma}$ could facilitate EMT and CSCs in BC cells, as evidenced by reductive E-cadherin expression and incremental Vimentin level [45]. In this course, ErbB2 and ErbB4 act as the major upstream receptor kinases. Both are capable to activate $\mathrm{p} 38 \mathrm{\gamma}$ through ubiquitin-proteasome-dependent degradation and the degradation of GATA3 is caused subsequently. This suppression causes the up-regulation of $\mathrm{miR}-200 \mathrm{~b}$ and down-regulates the expression of Suz12, eventually leading to EMT and invasiveness of BC [45].

Distinguishingly, among all types of $\mathrm{BC}$, triple negative $B C$ (TNBC) tends to have the worst prognosis due to the lack of therapeutic targeting of ER, progesterone receptor (PR), and epidermal growth factor receptor 2 (HER-2) [46]. Meanwhile TNBC is prominently heterogeneous but abundant among the CSCs population [47]. Qi et al. point out p38 $\gamma$ activates Nanog transcription by forming a polyprotein complex with c-Jun/Hsp90 on the Nanog promoter and activates the expression of transcription factors such as Sox 2 and Oct3/4 to stimulate CSCs amplification in TNBC cells. These consequences further suggest that the coalescence of Hsp90 inhibitors with the non-toxic p38 $\gamma$ inhibitor PFD may even be more efficacious in treating TNBC by destroying functional protein complexes, thereby co-consuming the CSCs population [48].

\section{Squamous Cell Carcinoma}

Lack of $\mathrm{p} 38 \mathrm{\gamma}$ makes the incidence and number of tumors per mouse lower than that of wild-type mice [21]. Researchers demonstrated that $\mathrm{p} 38 \gamma$ and $\mathrm{p} 38 \delta$ are concerned with the production of cytokines such as IL-6, STAT3, and TNF-a in the skin, contributing to

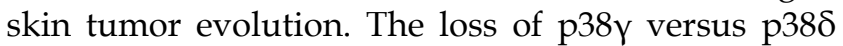
abrogated tumorigenesis in human epidermoid carcinoma A431 cells [21].

In the case of esophageal squamous cell carcinoma (ESCC), stable down-regulation of p38y can prevent tumorigenesis in a nude mouse model of ESCC cell xenotransplantation [49]. In addition, p38y can promote the movement and proliferation, and prevent the apoptosis of ESCC cells in vitro [49]. Similarly, higher p38 $\gamma$ expression is also observed in head and neck squamous cell carcinoma cells [20].

\section{Hepatocellular Carcinoma}

p38 $\gamma$ is immediately related to the expression of ACTA2 and COL1A [31]. Both these two genes encode actin and collagen respectively, which are fibrotic markers that commonly used before liver cancer develops, indicating the HCC with p38y elevation is easier to migrate and deteriorate [31].

Experiments by Tomas-Loba et al. showed that p38y staining in human HCC biopsy tissues is much stronger than in non-tumor tissues and p38 $\gamma$ knockdown weakened proliferation and colonization in HCC cell lines [31]. In addition, HCC in mice lacking $\mathrm{p} 38 \gamma$ in hepatocytes (AlbCre-p38 $\gamma$ mice) is observed to be strongly controlled. Compared with control (AlbCre+/-) mice, their tumors were smaller, fewer, and survived longer [31]. Albcre-p38 $\gamma$ mice additionally showed the ability to prevent liver cancer induced by carbon tetrachloride or high-fat diet [50]. As a cyclin-dependent kinase (CDK)-like kinase, p38 y exhibits a sort of substrate specificity and inhibition sensitivity analogical to conventional CDKs, which is manifested as phosphorylate the $\mathrm{C}$ terminus of retinoblastoma tumor suppressor protein $(\mathrm{Rb})$ to promote liver proliferation [16, 31]. p38 $\gamma$ may stand for a unique kinase that activates the non-classical CDK pathway in a cell cycle protein-independent manner when the CDK-cyclin complex is low, thus initiating the cell cycle and allowing the cells to escape from quiescence under stress stimulation. Collectively, these evidences undoubtedly confirm that $\mathrm{p} 38 \mathrm{\gamma}$ is necessary for $\mathrm{Rb}$-dependent cell cycle progression and liver tumorigenesis, supporting the potential of $\mathrm{p} 38 \mathrm{\gamma}$ as a target for HCC treatment.

\section{Colorectal Cancer}

Loss of $\mathrm{p} 38 \mathrm{\gamma}$ significantly inhibits cell growth, proliferation, and migration in HT3-29 cells and primary human CRC cells, while suppressing apoptosis. In particular, $\mathrm{p} 38 \mathrm{\gamma}$ standards are observably increased in human CRC tissues in terms of the surrounding normal epithelial tissues [34]. Consistent with previous research, $\mathrm{p} 38 \mathrm{\gamma}$ deletion also 
results in decreased $\mathrm{Rb}$ phosphorylation and cyclin (E1/A) expression, both of which are augmented as p38y highly expressed in CRC cells [34].

As an important regulatory factor, $\mathrm{p} 38 \mathrm{\gamma}$ promotes CRC tumorigenesis by transmitting exogenous and endogenous signals. For example, conditional p38y knockout of intestinal epithelial cells (IECs) reduces the expression of proinflammatory cytokine and $\beta$-Catenin/Wnt pathway activity in colon tissues, successfully linking inflammation with CRC [51]. Chronic application of PFD can alleviate CRC induced by inflammation in mice, but no effect in mice with conditional p38y knockout in IECs [51]. In addition, in the AOM/DSS mouse model, p38y deficiency also attenuates the generation of CRC [51].

The Ras-dependent mechanism is an important part of the occurrence of CRC [52]. PTPH1 acts as an exclusive p38 subtype-specific phosphatase known to interact through PDZ binding [53]. Hou et al. found through experiments that $\mathrm{p} 38 \mathrm{\gamma}$ can directly phosphorylate PTPH1 to promote the growth of Ras conversion and Ras-dependent CRC [52]. In human CRC tissues, the transcription factor c-Jun was activated by phosphorylated $\mathrm{p} 38 \mathrm{\gamma}$ through its C-terminus, and then the activated c-Jun recruited p38 $y$ as a cofactor to the matrix metalloproteinase 9 (MMP9) promoter, inducing its transactivation and ultimately promoting CRC cell invasiveness [3].

\section{Pancreatic Ductal Adenocarcinoma}

p38 $\mathrm{y}$ has been reported to link K-Ras signaling and the Warburg effect by means of phosphofructokinase-2/fructose-2,6-bisphosphatase 3 (PFKFB3) and glucose transporter 2 (GLUT2). It's crucial for aerobic glycolysis and pancreatic tumorigenesis [16]. The $\mathrm{p} 38 \mathrm{\gamma}$ activation-dependent phosphorylation and stabilization of PFKFB3 and its interaction with GLUT2 constitute a ternary complex, which can effectively boost the occurrence of aerobic enzymes and pancreatic ductal adenocarcinoma (PDAC) via their regionally elevated concentrations. The course of cell cycles from the G1 to $S$ phase in p38y silenced pancreatic ductal epithelial cells (KPC) cells has been found to be blocked compared with KPC and associated with lower levels of CDK6 (cyclin dependent kinase 6) and $\mathrm{p}-\mathrm{Rb}$ proteins [16]. The function of $p 38 \gamma$ in the G1/S transition may be critical for stimulating cell proliferation and cell motility, activating glycolysis, promoting inflammatory responses as well as the development and growth of PADC [16, 54]. In addition, a novel therapeutic strategy established by PFD and the PFKFB3 inhibitor PFK15 can inhibit glucose uptake, reduce lactic acid secretion, and suppress PDAC growth [16].

\section{Glioma}

p38 $\gamma$ defect results in lessened proliferation and enhancive apoptosis of glioma cells [55]. With the decrease of $p 38 \gamma$, the expression of human telomerase reverse transcriptase (hTERT) and telomerase show a corresponding decline, while the expression of Caspase-3/9 increases [55]. Glioblastoma (GBM) is the most malignant and common glioma [56]. Previous studies have shown that the transcription factor REST (repressor element 1-silencing transcription factor) may be present as a cancer gene in GBM [37]. As a downstream gene of REST, p38y can be inhibited by the complex formed by REST and its cofactors, which may trigger the transition of G1-S phase in cell cycle. The inhibition of p38y by REST may eventually lead to the proliferation and migration of GBM cells, which partially proves the tumor suppressor effect of $\mathrm{p} 38 \mathrm{\gamma}$ [37]. In glioma of the entire nervous system, the controversial results shown by $\mathrm{p} 38 \mathrm{\gamma}$ so far may be due to different experimental conditions and experimental designs. Therefore, appropriate experiments are needed to verify the fundamental role of $p 38 \mathrm{y}$ in glioma next.

\section{Cutaneous T-cell Lymphoma}

p38 $\gamma$ is likely to be an important breakthrough in improving the level of CTCL diagnosis and treatment in the future. It selectively increases its expression to promote cell activity in CTCL patients and cell lines [4]. Meanwhile multi-kinase inhibitor F7/PIK75 could efficaciously restrain p38y enzymatic activity and the production of its phosphorylated substrate pDLDH1 in CTCL cells and mouse xenografts [4].

Sézary syndrome (SS) is a form of CTCL that appears on the skin. Based on different investigations in vitro, for instance, the expression of $\mathrm{p} 38 \mathrm{y}$ is higher in $\mathrm{CD}^{+} \mathrm{T}$ cells in patients with Sézary syndrome than in healthy blood donors [57]. In 2018, a private non-profit clinical research center and hospital called City of Hope filed for a patent, which included a new p38y inhibitor and its application in the treatment of CTCL [57]. The lead compound inhibitor in this patent has ATP competitive inhibitory effect on $p 38 \gamma$, and this compound shows dose-dependent inhibitory effect on tumor growth in CTCL xenograft model [57]. The additional library of EMD Biosciences screens 260 kinase inhibitors, including two novel potential p38 $\mathrm{\gamma}$ inhibitors, and shows a typical-I p38 inhibitor binding schema to determine that $\mathrm{p} 38 \gamma$ is a potential objective for the therapy of CTCL [57].

\section{Osteosarcoma}

miR-187 is confirmed to be the direct upstream molecule of p38 $\mathrm{\gamma}$ in osteosarcoma cells [58]. The low 
level of miR-187 is significantly related to staging, lymph node metastasis and deep stromal infiltration. By inhibiting $\mathrm{p} 38 \mathrm{\gamma}$, it reduces the biological behaviors of osteosarcoma cells like cell proliferation, migration and invasion. Correspondingly the reverse expression of miR-187 and p38 $\gamma$ in specimens of OS patients further confirmed this process [58].

Notably, the main cause of OS abnormal growth is the uncontrolled cell cycle and cell division [59]. p38y can also play a cancer-promoting part in OS by stimulating $\mathrm{Rb}$ phosphorylation and cyclin E1/A expression and regulating G1-S phase transition [36]. Accordingly, present study shows that mRNA and protein expression of $\mathrm{p} 38 \mathrm{\gamma}$ in human OS tissue and primary OS cells were significantly higher than that in human primary osteoblasts and OB-6 osteoblastic cells [36].

\section{Renal Cell Carcinoma}

p38 $\gamma$ can analogously serve as a novel cyclin dependent kinase (CDK)-like kinase, which can phosphorylate $\mathrm{Rb}$ at the molecular level and regulate the cell cycle protein cyclin E1/A expression, ultimately promoting the progression of RCC cells [35]. Correspondingly, ectopic overexpression of p38y promoted the growth, proliferation and migration of 786-O cells and primary RCC cells [35]. Besides, it manifested an increase of $\mathrm{p} 38 \mathrm{\gamma}$ mRNA and protein degree in human renal cell carcinoma instead of adjacent tissues [35]. The results of Aguilar et al. showed that the level of p38y in the kidney increased gradually after 1 and 2 months of cancer induction with ferric nitrilotriacetate (FeNTA) [60]. In this case, with the evolvement of cancer, the level of p38y in the tumor even reached more than 30 times of that in the normal kidney [60].

A

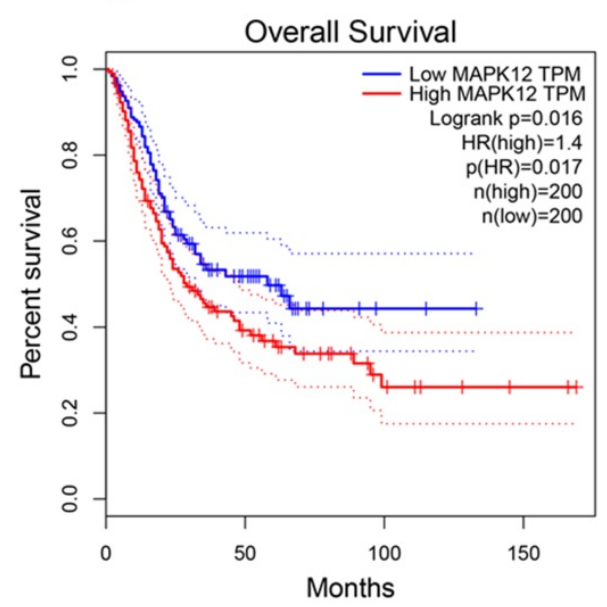

\section{Non-Small Cell Lung Cancer}

Cancer stem cells (CSCs) are the main source of tumorigenesis and recurrence and participate in p38y-mediated inhibition of NSCLC. Activated p38y can mediate the ubiquitination and degradation of stem cell proteins such as Sox2, Oct4 and Nanog by means of activating the downstream kinase MK2 to phosphorylate Hsp27, thereby inhibiting the tumor stem cell characteristics and tumor initiation ability of NSCLC cells [12]. Conversely, a significant increase in p38 $\gamma$ is associated with carcinogenicity of NSCLC, but not with stage, tumor, or response to treatment [61]. As of yet, the research of p38 $\gamma$ in NSCLC is limited, especially when the current opinions are contradictory without the specific role and mechanism of $\mathrm{p} 38 \mathrm{\gamma}$.

\section{Bladder Cancer}

Kaplan Meier analysis shows that the expression of $\mathrm{p} 38 \mathrm{\gamma}$ in bladder cancer is significantly different $(\mathrm{P}<$ $0.05)$, and the prognosis of patients with high expression of $\mathrm{p} 38 \gamma$ is often gravely unfavorable [62]. p38 $\gamma$ may have the ability to become a prognostic marker and therapeutic target of bladder cancer. We used GEPIA (Gene Expression Profile Interactive Analysis): http://gepia.cancer-pku.cn/ to conduct a correlation analysis and found that the high expression of $\mathrm{p} 38 \mathrm{\gamma}$ in bladder cancer was negatively bound up with the overall survival of patients. Additionally, $\mathrm{p} 38 \mathrm{\gamma}$ expression in different pathological stages (stage II, stage III, and stage IV) of bladder cancer had conspicuous significance (Figure 2). However, the mechanism of p38y in bladder cancer is still obscure in experiments, consequently p38y may be used as a potential research target.

B

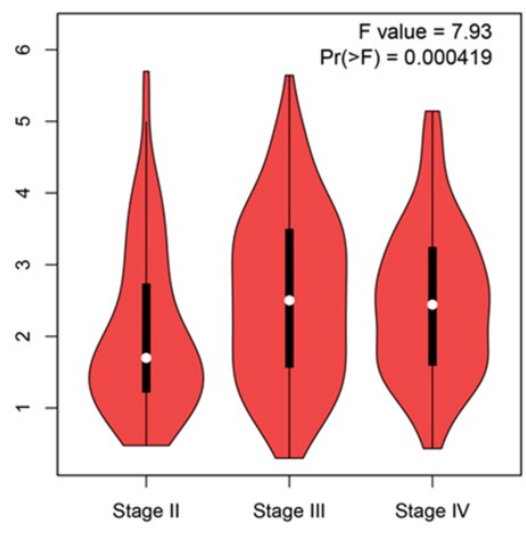

Figure 2. Expression of $\mathrm{p} 38 \mathrm{y}$ in different survival rates and different stages of bladder cancer. (A) Correlation analysis between $\mathrm{p} 38 \mathrm{y}$ and overall survival in bladder cancer $(p=0.017)$; (B) The expression of $p 38 y$ in different clinical stages of bladder cancer $(p=0.000419)$. 


\section{The latent clinical value of $\mathrm{p} 38 \mathrm{y}$ in human carcinoma}

The early detection and diagnosis of cancer is crucial for patients. Sensitive tumor biomarkers are capable of reflecting the molecular differences of certain exceptive cancers when they occur at an early stage, which is not only propitious to inchoate diagnosis, but also advantageous to the screener and prognostic judgment of synthetical treatment tactics. Coincidentally, relevant studies have shown that the expression of $\mathrm{p} 38 \gamma$ is closely bound up with the depth of invasion, clinical stage, metastasis, and chemosensitivity. This section summarizes reports of $\mathrm{p} 38 \gamma$ as a biological marker for cancer diagnosis and prognosis and also summarizes the effect of $p 38 \gamma$ on the sensitivity of tumor chemotherapy.

Literatures have demonstrated the value of $\mathrm{p} 38 \mathrm{\gamma}$ in diagnosis. For example, researchers use immunohistochemistry with tissue microarray and performed a statistical analysis of its clinicopathological significance [49]. The expression of $\mathrm{p} 38 \mathrm{\gamma}$ in ESCC is significantly different between clinical stage $(\mathrm{P}=0.017)$, lymph node metastasis $(\mathrm{P}=0.008)$ and tumor volume $(\mathrm{P}=0.017)[49]$. In terms of prognosis, no conspicuous discrepancy is observed between patients with different levels of p38y expression $(\mathrm{P}=$ 0.667) in ESCC [49]. In a HCC cohort composed of 372 HCC patients, the Kaplan-Meier survival curve of patients stratifies by $\mathrm{p} 38 \mathrm{\gamma}$ expression shows that $\mathrm{p} 38 \mathrm{\gamma}$ overexpression is associated with poor prognosis of HCC patients [31]. Among 43 samples of BC patients whose molecular subtypes are analyzed by PAM50 before, high expression of $\mathrm{p} 38 \gamma$ is accompanied by the obvious enrichment of $\mathrm{BC}$ basal-like subtypes $(\mathrm{P}=$ 0.018) [41]. The expression of $p 38 \gamma$ is also analyzed in 118 BC patients, including survival data. The results show that there is a consanguineous correlation between the higher expression of $\mathrm{p} 38 \mathrm{y}$ and the lower overall survival rate $(P=0.013)$, suggesting that the expression of $\mathrm{p} 38 \mathrm{\gamma}$ is of great significance for the prognosis of BC patients [41].

Chemotherapy still consists of the mainstay in cancer treatment. It has been reported that p38y can transduce signals related to three widely used chemotherapy drugs, cisplatin, etoposide and tamoxifen [63].

\section{Outlook}

A serry of studies showed the impact of p38 $\gamma$ on carcinogenesis, invasion, maintenance and resistance to chemotherapy. The $\mathrm{p} 38 \mathrm{\gamma}$ signal pathway leads to incremental expression of multiple downstream targets including transcriptional regulators. The transcription targets regulated by $\mathrm{p} 38 \mathrm{\gamma}$ may be either oncogenes or tumor suppressors, determined by different organs and tissue. Among the associated research of $\mathrm{p} 38 \mathrm{\gamma}$ in cancer, conflicting data in specific cancer types indicate that the full role of $\mathrm{p} 38 \mathrm{\gamma}$ is unclear, and further research is required to adequately comprehend the ponderance of $\mathrm{p} 38 \mathrm{\gamma}$ in human pathology. This section summarizes the update and the prospect of $\mathrm{p} 38 \gamma$ in life sciences.

Drugs that directly target DNA topoisomerase II a (Topo IIa or Topo II) represent a vital class of anti-tumor drugs in clinic. It has been proved that p38y can actively regulate the signal transduction of the drug-Topo II and can improve the therapeutic activity of Topo II drugs [9]. In this process, p38 $\gamma$ is specifically activated by Topo II drugs, and thereafter activated p38y phosphorylates Topo II at Ser-1524, resulting in stronger stability and growth inhibition. The combination of p38 $\gamma$ and Topo II drugs may have clinical value for tumor disease. So far, one $\mathrm{p} 38 \mathrm{\gamma}$ inhibitor PFD was applied in clinical. It has been experimentally proven in vivo that its inhibitory effect on $\mathrm{p} 38 \mathrm{\gamma}$ requires a high daily dose $(500 \mathrm{mg} / \mathrm{kg} /$ day in drinking water) [51], which raises doubts about its clinical value in anti-cancer fields. Conversely, among more than 20 p38 family candidates in clinical trials [57], for instance, combined p38 $\gamma$ and p38 inhibitor (BIRB796) against IFN- $\gamma$ [64], multi-kinase inhibitor F7/PIK75 which effectively against $\mathrm{p} 38 \gamma$ for CTCL treatment [4], pirfenidone is the exclusive drug targeting $\mathrm{p} 38 \mathrm{\gamma}$ clinically for anti-fibrosis [16]. Additionally, the Drugbank platform showed that the current experimental and investigational drugs targeting $\mathrm{p} 38 \mathrm{y}$ include Phosphonothreonine, Phosphoaminophosphonic Acid-Adenylate Ester, CEP-1347 and KC706 [65]. A number of pre-clinical and clinical trials were needed to explore more effective $\mathrm{p} 38 \mathrm{y}$ inhibitors.

The relationship between intestinal microbes and cancer is attracting immense attention in gastrointestinal cancer and cancers of other metabolic organs [66-68]. Imidazole propionate is a histidinederived metabolite produced by microorganisms. In 2018, research by Ara Koh et al. showed that imidazole propionate could activate $\mathrm{p} 38 \mathrm{\gamma}$ to promote the phosphorylation of p62 and activate the mechanical target of rapamycin complex 1 (mTORC1). The demonstration of this impairs insulin signal transduction at the level of insulin receptor substrates. This interaction between the intestinal flora metabolites and p38y will eventually lead to the development of metabolic diseases, and potentially linked to the pathological conditions of cancer caused by the continuous activation of mTORC1 [68]. On the other hand, the signal adapter p62 is a multi-domain protein concerned with the activation of the 
transcription factor NF-kB; the activity of p62 is closely related to the external apoptosis pathway, as well as autophagy, a key factor in tumorigenesis [69]. In 2020, further research by Ara Koh et al. found that the microbial metabolite imidazole propionate can reduce the effectiveness of metformin by interacting with the $\mathrm{p} 38 \mathrm{\gamma} / \mathrm{AKT} / \mathrm{AMPK}$ metformin signaling pathway [70]. Although metformin is a major impactful drug for the treatment of type 2 diabetes, epidemiological studies have demonstrated a correlation between the application of metformin and the beneficial effects of cancer therapy [71]. In summary, p38y may play as a key molecule in microbial metabolism that affects the effect of metformin on cancer. We hypothesize that the intestinal flora may regulate the pathogenesis of cancer by influencing $\mathrm{p} 38 \mathrm{\gamma}$.

Given that cancer is a genetic disease, gene therapy is expected to fight cancer from within with minimal side effects, and there is evidence that CRISPR-Cas9 can correct cancer-causing genome aberrations and induce the expression of tumor suppressor genes to achieve cancer gene therapy [72]. Using CRISPR-Cas9 inhibits oncogenes such as p38y gene in some tumors, which may become a promising anti-cancer strategy. Researchers have used CRISPRCas9-induced $\mathrm{p} 38 \mathrm{\gamma}$ knockout to inhibit the in vitro progression of human OS cells and the growth, proliferation and migration of human CRC cells and human RCC cells and induce significant apoptosis [34-36]. On the other hand, it is reported that CRISPRCas9 can be used as a powerful tool to manipulate epigene regulation to treat cancer and reshape abnormal epigenetic patterns and can selectively and genetically alter gene expression [73]. For example, nuclease-inactivated dCas9 is coalesced with certain epigenetic modifiers such as acetyltransferases to shape a compound, which enters the target site under the lead of a single guide RNA (sgRNA), and finally exerts an epigenetic regulation effect. This process is also known as epigenome editing [74, 75]. p38 $\gamma$ serves as the part of the corresponding gene expression product of epigenetic modification, may also be adaptive for epigenome editing therapy.

Immunotherapy has become a considerable part that cannot be ignored, including immunosuppressants targeting PD-1 for T cells and PD-L1 for tumor cells in recent years. The most cutting-edge Car- $T$ therapy is also getting more attention. The efficacy of immunotherapy varied in individual patients and cancer diseases. The level of PD-L1 is the most influential factor to affect the anti-tumor effect of immunotherapy. Immune checkpoint inhibitors (ICIs) targeting PD-1 or PD-L1 have already greatly improved the prognosis in different cancer types and prolong the patients' survival as well as quality of life. The approach that uses a panel of biomarkers to guide patient selection may provide an opportunity to improve patient outcomes relative to a single biomarker such as PD-L1 [76]. Based on the studies above, it may be feasible to combine the ICIs prediction biomarker represented by PD-L1 with p38y.

One of the focus of anti-tumor immunotherapy is to activate $\mathrm{CD}^{+} \mathrm{T}$ cells and maximize the function of $\mathrm{CD}^{+} \mathrm{T}$ cells. $\mathrm{CD} 8{ }^{+} \mathrm{T}$ cells play a tumorspecific killing effect with the assistance of $\mathrm{CD} 4^{+} \mathrm{T}$ cells. As $\mathrm{CD}^{+} \mathrm{T}$ cells continues to function, immune checkpoints gradually appear on the surface of cell membranes, and bind to receptors from cancer cells, which eventually leads to the death of $\mathrm{CD}^{+} \mathrm{T}$ cells. This is an immunosuppressive process and is also a major cause of immune escape from tumors. p38 $\mathrm{y}$ plays a basilic role in the development and differentiation of $\mathrm{T}$ lymphocytes. The deletion of $\mathrm{p} 38 \mathrm{\gamma}$ can facilitate the positive selection of thymocytes from $\mathrm{CD}^{+}{ }^{+} \mathrm{CD} 8^{+}$double-positive cells to $\mathrm{CD}^{+}$or $\mathrm{CD}^{+}$ single-positive cells [77]. Therefore, the clinical use of p38 $\gamma$ inhibitors may coalesce $\mathrm{T}$ cell immunotherapy with $\mathrm{p} 38 \mathrm{\gamma}$ itself as an oncogene to develop more effective anti-cancer methods.

\section{Conclusion}

While the past decade has witnessed the rapid development of cancer researches, cancer treatment methods are nothing new. Ample evidence proved that $\mathrm{p} 38 \mathrm{\gamma}$ possesses a basilic function in the oncogenesis and cancer development, including proliferation, EMT, invasion, metastasis. This heralds that $\mathrm{p} 38 \gamma$ may play an excellent function in diagnosis, treatment, and prognostic indicators in related tumor types. Despite the role of $\mathrm{p} 38 \mathrm{y}$ inhibitors in clinical tumors is not prominent, there is plenty of room for perfection. We summarized the primary impact of p38 $\gamma$ at different stages of a variety of tumors through several classic signal transduction pathways (Figure 3 ), besides foremost expectations. The future research orientation of $\mathrm{p} 38 \mathrm{\gamma}$ may be based on large-scale clinical trials and further in-depth mechanisms. p38y will definitely elicit audacious threads for comprehending and treating cancer.

\section{Acknowledgements}

\section{Authors' contributions}

HW and WTX design work, article analysis and key revisions, graphics and table design. WTX, RL, $\mathrm{YD}$ and $\mathrm{SCH}$ drafted the manuscript, review the related articles. WTX, RL, YD, SCH and HKD analyzed of final approval. 


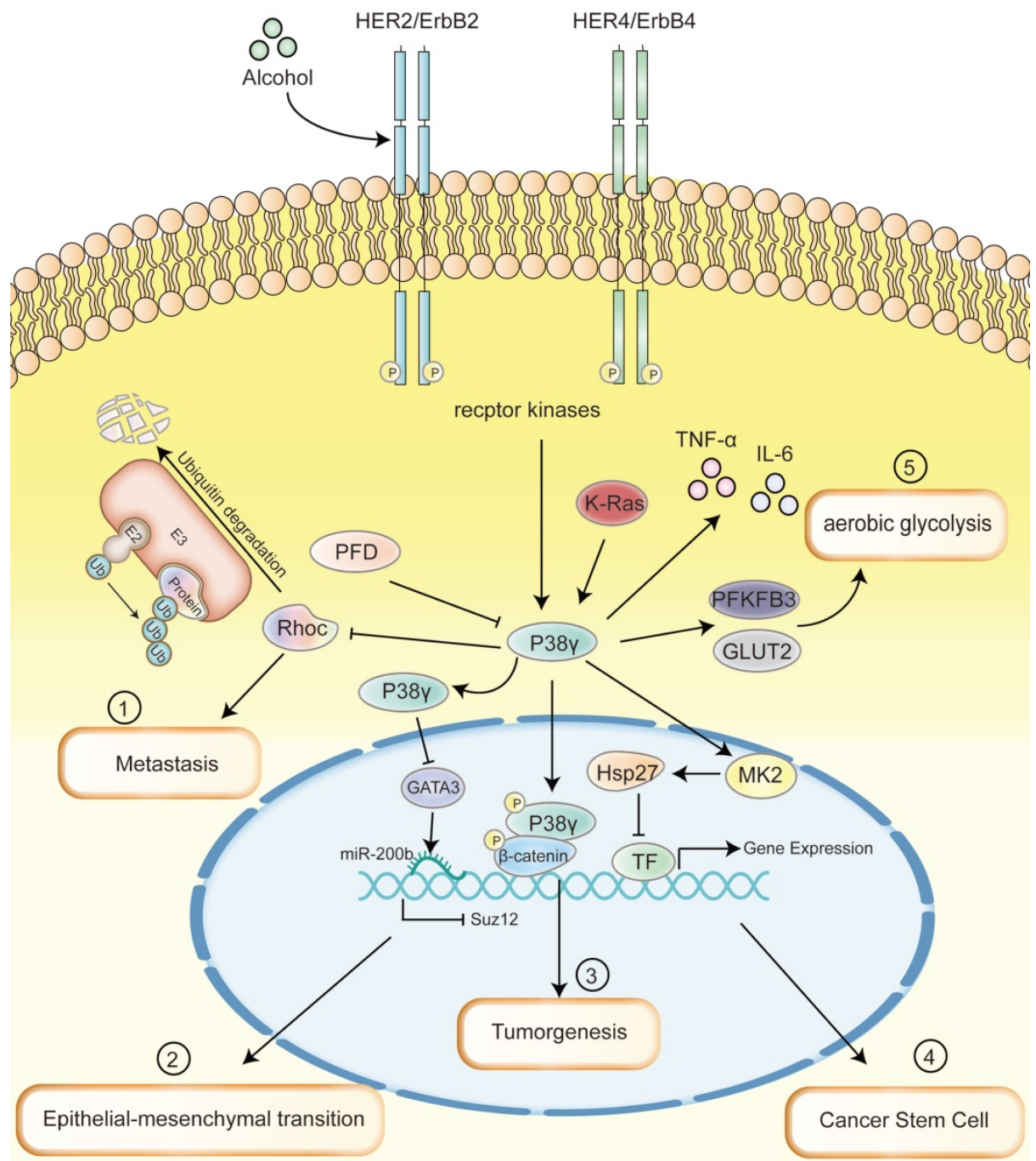

Figure 3. Multiple signaling pathways that p38y mainly involved in a range of cancer diseases. (1) $p 38 y$ regulates the ubiquitination of Rho $C$ related to metastasis to control cell movement and promote the metastasis of BC. (2) ErbB2/ErbB4/p38y/GATA3/miR-200b/Suz12 pathway promotes the acquisition of the EMT phenotype, which may mediate the aggressiveness of BC. (3) The silencing of $\mathrm{p} 38 \mathrm{y}$ in intestinal epithelial cells can reduce the expression of pro-inflammatory cytokines and the activity of $\beta$-Catenin/Wnt pathway in colon tissue, thereby successfully linking inflammation with CRC, leading to tumorigenesis of CRC. (4) Upregulated p38y can phosphorylate Hsp27 protein by activating the downstream kinase MK2, which binds to transcription factor proteins such as Sox2, Oct4, and Nanog to cause ubiquitination and degradation, thereby inhibiting the cancer stem cell characteristics and tumor initiating ability of NSCLC. (5) As the upstream of p38y, K-Ras promotes the activation of p38y, increases the expression of PFKFB3 and GLUT2, thus encourages aerobic glycolysis and facilitates the occurrence of PDAC. Throughout the process, cytokines such as TNF- $\alpha$ and IL-6 produced by P38y link the inflammation and cancer; PFD is able to specifically inhibit the phosphorylation of $\mathrm{P} 38 \mathrm{y}$.

\section{Competing Interests}

The authors have declared that no competing interest exists.

\section{References}

1. Mertens S, Craxton M, Goedert M. SAP kinase-3, a new member of the family of mammalian stress-activated protein kinases. FEBS Lett. 1996; 383: 273-6.

2. Qi X, Pohl N M, Loesch M, Hou S, Li R, Qin J Z, et al. p38alpha antagonizes p38gamma activity through c-Jun-dependent ubiquitin-proteasome pathways in regulating Ras transformation and stress response. J Biol Chem. 2007; 282: 31398-408.

3. Loesch M, Zhi H Y, Hou S W, Qi X M, Li R S, Basir Z, et al. p38gamma MAPK cooperates with c-Jun in trans-activating matrix metalloproteinase 9. J Biol Chem. 2010; 285: 15149-58.

4. Zhang X H, Nam S, Wu J, Chen C H, Liu X, Li H, et al. Multi-Kinase Inhibitor with Anti-p38gamma Activity in Cutaneous T-Cell Lymphoma. J Invest Dermatol. 2018; 138: 2377-2387.

5. Cuadrado A, Nebreda A R. Mechanisms and functions of p38 MAPK signalling. Biochem J. 2010; 429: 403-17.

6. Genera M, Samson D, Raynal B, Haouz A, Baron B, Simenel C, et al. Structural and functional characterization of the PDZ domain of the human phosphatase
PTPN3 and its interaction with the human papillomavirus E6 oncoprotein. Sci Rep. 2019; 9: 7438.

7. Sabio G, Cerezo-Guisado M I, Del Reino P, Inesta-Vaquera F A, Rousseau S, Arthur J S, et al. p38gamma regulates interaction of nuclear PSF and RNA with the tumour-suppressor hDlg in response to osmotic shock. J Cell Sci. 2010; 123: 2596-604.

8. Escos A, Risco A, Alsina-Beauchamp D, Cuenda A. p38gamma and p38delta Mitogen Activated Protein Kinases (MAPKs), New Stars in the MAPK Galaxy. Front Cell Dev Biol. 2016; 4: 31

9. Qi X, Hou S, Lepp A, Li R, Basir Z, Lou Z, et al. Phosphorylation and stabilization of topoisomerase IIalpha protein by p38gamma mitogenactivated protein kinase sensitize breast cancer cells to its poisons. J Biol Chem. 2011; 286: 35883-90.

10. Tang J, Qi X, Mercola D, Han J, Chen G. Essential role of p38gamma in K-Ras transformation independent of phosphorylation. J Biol Chem. 2005; 280: 23910-7.

11. Qi X, Tang J, Loesch M, Pohl N, Alkan S, Chen G. p38gamma mitogenactivated protein kinase integrates signaling crosstalk between Ras and estrogen receptor to increase breast cancer invasion. Cancer Res. 2006; 66: 7540-7.

12. Fang $\mathrm{Y}$, Wang J, Wang G, Zhou C, Wang P, Zhao S, et al. Inactivation of p38 MAPK contributes to stem cell-like properties of non-small cell lung cancer. Oncotarget. 2017; 8: 26702-26717.

13. Degirmenci U, Wang M, Hu J. Targeting Aberrant RAS/RAF/MEK/ERK Signaling for Cancer Therapy. Cells. 2020; 9 . 
14. Cargnello M, Roux P P. Activation and function of the MAPKs and their substrates, the MAPK-activated protein kinases. Microbiol Mol Biol Rev. 2011; 75: $50-83$.

15. Ono K, Han J. The p38 signal transduction pathway: activation and function. Cell Signal. 2000; 12: 1-13.

16. Wang F, Qi X M, Wertz R, Mortensen M, Hagen C, Evans J, et al. p38gamma MAPK Is Essential for Aerobic Glycolysis and Pancreatic Tumorigenesis. Cancer Res. 2020; 80: 3251-3264.

17. Han J, Lee J D, Bibbs L, Ulevitch R J. A MAP kinase targeted by endotoxin and hyperosmolarity in mammalian cells. Science. 1994; 265: 808-11.

18. Cuenda A, Rousseau S. p38 MAP-kinases pathway regulation, function and role in human diseases. Biochim Biophys Acta. 2007; 1773: 1358-75.

19. Meng $\mathrm{F}, \mathrm{Wu} \mathrm{G}$. Is p38gamma MAPK a metastasis-promoting gene or an oncogenic property-maintaining gene? Cell Cycle. 2013; 12: 2329-30.

20. Sahu V, Nigam L, Agnihotri V, Gupta A, Shekhar S, Subbarao N, et al. Diagnostic Significance of p38 Isoforms (p38alpha, p38beta, p38gamma, p38delta) in Head and Neck Squamous Cell Carcinoma: Comparative Serum Level Evaluation and Design of Novel Peptide Inhibitor Targeting the Same. Cancer Res Treat. 2019; 51: 313-325.

21. Zur R, Garcia-Ibanez L, Nunez-Buiza A, Aparicio N, Liappas G, Escos A, et al. Combined deletion of p38gamma and p38delta reduces skin inflammation and protects from carcinogenesis. Oncotarget. 2015; 6: 12920-35.

22. Risco A, Cuenda A. New Insights into the p38gamma and p38delta MAPK Pathways. J Signal Transduct. 2012; 2012: 520289

23. Kastan M B, Bartek J. Cell-cycle checkpoints and cancer. Nature. 2004; 432: 316-23.

24. Chen X, Du Y, Liu Y, He Y, Zhang G, Yang C, et al. Hyaluronan arrests human breast cancer cell growth by prolonging the G0/G1 phase of the cell cycle. Acta Biochim Biophys Sin (Shanghai). 2018; 50: 1181-1189.

25. Gubern A, Joaquin M, Marques M, Maseres P, Garcia-Garcia J, Amat R, et al. The N-Terminal Phosphorylation of RB by p38 Bypasses Its Inactivation by CDKs and Prevents Proliferation in Cancer Cells. Mol Cell. 2016; 64: 25-36.

26. Jin S, Tong T, Fan W, Fan F, Antinore M J, Zhu X, et al. GADD45-induced cell cycle G2-M arrest associates with altered subcellular distribution of cyclin B1 and is independent of p38 kinase activity. Oncogene. 2002; 21: 8696-704.

27. Liu Y, Chang Y, Cai Y. circTNFRSF21, a newly identified circular RNA promotes endometrial carcinoma pathogenesis through regulating miR-1227-MAPK13/ATF2 axis. Aging (Albany NY). 2020; 12: 6774-6792.

28. Conrad P W, Rust R T, Han J, Millhorn D E, Beitner-Johnson D. Selective activation of p38alpha and p38gamma by hypoxia. Role in regulation of cyclin D1 by hypoxia in PC12 cells. J Biol Chem. 1999; 274: 23570-6.

29. Wang X, McGowan C H, Zhao M, He L, Downey J S, Fearns C, et al. Involvement of the MKK6-p38gamma cascade in gamma-radiation-induced cell cycle arrest. Mol Cell Biol. 2000; 20: 4543-52.

30. Perdiguero E, Pillaire M J, Bodart J F, Hennersdorf F, Frodin M, Duesbery N S, et al. Xp38gamma/SAPK3 promotes meiotic $G(2) / M$ transition in Xenopus oocytes and activates Cdc25C. EMBO J. 2003; 22: 5746-56.

31. Tomas-Loba A, Manieri E, Gonzalez-Teran B, Mora A, Leiva-Vega L, Santamans A M, et al. p38gamma is essential for cell cycle progression and liver tumorigenesis. Nature. 2019; 568: 557-560

32. Meng F, Zhang H, Liu G, Kreike B, Chen W, Sethi S, et al. p38gamma mitogen-activated protein kinase contributes to oncogenic properties maintenance and resistance to poly (ADP-ribose)-polymerase-1 inhibition in breast cancer. Neoplasia. 2011; 13: 472-82.

33. Kukkonen-Macchi A, Sicora O, Kaczynska K, Oetken-Lindholm C, Pouwels J, Laine L, et al. Loss of p38gamma MAPK induces pleiotropic mitotic defects and massive cell death. J Cell Sci. 2011; 124: 216-27.

34. Su C, Sun Q, Liu S, Wang H, Feng L, Cao Y. Targeting p38gamma to inhibit human colorectal cancer cell progression. Biochem Biophys Res Commun. 2019; 517: 172-179.

35. Chen X F, Pan Y S, Zheng B, Lu Q. p38gamma overexpression promotes renal cell carcinoma cell growth, proliferation and migration. Biochem Biophys Res Commun. 2019; 516: 466-473.

36. Shi C, Cheng W N, Wang Y, Li D Z, Zhou L N, Zhu Y C, et al. p38gamma overexpression promotes osteosarcoma cell progression. Aging (Albany NY). 2020; 12: 18384-18395.

37. Zhang D, Li Y, Wang R, Li Y, Shi P, Kan Z, et al. Inhibition of REST Suppresses Proliferation and Migration in Glioblastoma Cells. Int J Mol Sci. 2016; 17.

38. Qi X, Zhi H, Lepp A, Wang P, Huang J, Basir Z, et al. p38gamma mitogen-activated protein kinase (MAPK) confers breast cancer hormone sensitivity by switching estrogen receptor (ER) signaling from classical to nonclassical pathway via stimulating ER phosphorylation and c-Jun transcription. J Biol Chem. 2012; 287: 14681-91.

39. Suresh P S, Ma S, Migliaccio A, Chen G. Protein-tyrosine phosphatase H1 increases breast cancer sensitivity to antiestrogens by dephosphorylating estrogen receptor at Tyr537. Mol Cancer Ther. 2014; 13: 230-8.

40. Chen H, Wang X, Guo F, Li P, Peng D, He J. Impact of p38gamma mitogen-activated protein kinase (MAPK) on MDA-MB-231 breast cancer cells using metabolomic approach. Int J Biochem Cell Biol. 2019; 107: 6-13.

41. Rosenthal D T, Iyer H, Escudero S, Bao L, Wu Z, Ventura A C, et al. p38gamma promotes breast cancer cell motility and metastasis through regulation of RhoC GTPase, cytoskeletal architecture, and a novel leading edge behavior. Cancer Res. 2011; 71: 6338-49.
42. Xu M, Wang S, Ren Z, Frank J A, Yang X H, Zhang Z, et al. Chronic ethanol exposure enhances the aggressiveness of breast cancer: the role of p38gamma. Oncotarget. 2016; 7: 3489-505

43. Xu M, Ren Z, Wang X, Comer A, Frank J A, Ke Z J, et al. ErbB2 and p38gamma MAPK mediate alcohol-induced increase in breast cancer stem cells and metastasis. Mol Cancer. 2016; 15: 52.

44. Dongre A, Rashidian M, Reinhardt F, Bagnato A, Keckesova Z, Ploegh H L, et al. Epithelial-to-Mesenchymal Transition Contributes to Immunosuppression in Breast Carcinomas. Cancer Res. 2017; 77: 3982-3989.

45. Xu M, Wang S, Wang Y, Wu H, Frank J A, Zhang Z, et al. Role of p38gamma MAPK in regulation of EMT and cancer stem cells. Biochim Biophys Acta Mol Basis Dis. 2018; 1864: 3605-3617.

46. Kim C, Gao R, Sei E, Brandt R, Hartman J, Hatschek T, et al. Chemoresistance Evolution in Triple-Negative Breast Cancer Delineated by Single-Cell Sequencing. Cell. 2018; 173: 879-893 e13.

47. Tam W L, Lu H, Buikhuisen J, Soh B S, Lim E, Reinhardt F, et al. Protein kinase $\mathrm{C}$ alpha is a central signaling node and therapeutic target for breast cancer stem cells. Cancer Cell. 2013; 24: 347-64.

48. Qi X, Yin N, Ma S, Lepp A, Tang J, Jing W, et al. p38gamma MAPK Is a Therapeutic Target for Triple-Negative Breast Cancer by Stimulation of Cancer Stem-Like Cell Expansion. Stem Cells. 2015; 33: 2738-47.

49. Zheng S, Yang C, Liu T, Liu Q, Dai F, Sheyhidin I, et al. Clinicopathological significance of p38beta, p38gamma, and p38delta and its biological roles in esophageal squamous cell carcinoma. Tumour Biol. 2016; 37: 7255-66.

50. Gonzalez-Teran B, Matesanz N, Nikolic I, Verdugo M A, Sreeramkumar V, Hernandez-Cosido L, et al. p38gamma and p38delta reprogram liver metabolism by modulating neutrophil infiltration. EMBO J. 2016; 35: 536-52.

51. Yin N, Qi X, Tsai S, Lu Y, Basir Z, Oshima K, et al. p38gamma MAPK is required for inflammation-associated colon tumorigenesis. Oncogene. 2016; 35: 1039-48.

52. Hou S, Suresh P S, Qi X, Lepp A, Mirza S P, Chen G. p38gamma Mitogenactivated protein kinase signals through phosphorylating its phosphatase PTPH1 in regulating ras protein oncogenesis and stress response. J Biol Chem. 2012; 287: 27895-905.

53. Hou S W, Zhi H Y, Pohl N, Loesch M, Qi X M, Li R S, et al. PTPH1 dephosphorylates and cooperates with p38gamma MAPK to increase ras oncogenesis through PDZ-mediated interaction. Cancer Res. 2010; 70: 2901-10.

54. Icard $\mathrm{P}$, Fournel $\mathrm{L}, \mathrm{Wu} \mathrm{Z}$, Alifano $\mathrm{M}$, Lincet $\mathrm{H}$. Interconnection between Metabolism and Cell Cycle in Cancer. Trends Biochem Sci. 2019; 44: 490-501.

55. Yang K, Liu Y, Liu Z, Liu J, Liu X, Chen X, et al. p38gamma overexpression in gliomas and its role in proliferation and apoptosis. Sci Rep. 2013; 3: 2089.

56. Bi J, Chowdhry S, Wu S, Zhang W, Masui K, Mischel P S. Altered cellular metabolism in gliomas - an emerging landscape of actionable co-dependency targets. Nat Rev Cancer. 2020; 20: 57-70.

57. Haller $\mathrm{V}$, Nahidino $\mathrm{P}$, Forster M, Laufer S A. An updated patent review of $\mathrm{p} 38$ MAP kinase inhibitors (2014-2019). Expert Opin Ther Pat. 2020; 30: 453-466.

58. Cui C, Shi X. miR-187 inhibits tumor growth and invasion by directly targeting MAPK12 in osteosarcoma. Exp Ther Med. 2017; 14: 1045-1050.

59. Cheng L, Wang C, Jing J. Cell Cycle Kinases in Osteosarcoma: Potential for Therapeutic Intervention. Curr Pharm Des. 2016; 22: 4830-4834.

60. Aguilar-Alonso F A, Solano J D, Vargas-Olvera C Y, Pacheco-Bernal I, Pariente-Perez T O, Ibarra-Rubio M E. MAPKs' status at early stages of renal carcinogenesis and tumors induced by ferric nitrilotriacetate. Mol Cell Biochem. 2015; 404: 161-70.

61. Sahu V, Mohan A, Dey S. p38 MAP kinases: plausible diagnostic and prognostic serum protein marker of non-small cell lung cancer. Exp Mol Pathol. 2019; 107: 118-123.

62. Lin J, Yang J, Xu X, Wang Y, Yu M, Zhu Y. A robust 11-genes prognostic model can predict overall survival in bladder cancer patients based on five cohorts. Cancer Cell Int. 2020; 20: 402

63. Davis A P, Grondin C J, Johnson R J, Sciaky D, King B L, McMorran R, et al. The Comparative Toxicogenomics Database: update 2017. Nucleic Acids Res. 2017; 45: D972-D978

64. Yamaguchi R, Kawata J, Yamamoto T, Ishimaru Y, Sakamoto A, Ono T, et al. Mechanism of interferon-gamma production by monocytes stimulated with myeloperoxidase and neutrophil extracellular traps. Blood Cells Mol Dis. 2015; 55: 127-33

65. Wishart D S, Feunang Y D, Guo A C, Lo E J, Marcu A, Grant J R, et al. DrugBank 5.0: a major update to the DrugBank database for 2018. Nucleic Acids Res. 2018; 46: D1074-D1082.

66. Gaines S, van Praagh J B, Williamson A J, Jacobson R A, Hyoju S, Zaborin A, et al. Western Diet Promotes Intestinal Colonization by Collagenolytic Microbes and promotes Tumor Formation after Colorectal Surgery. Gastroenterology. 2020; 158: 958-970 e2

67. Dempsey J, Zhang A, Cui J Y. Coordinate regulation of long non-coding RNAs and protein-coding genes in germ-free mice. BMC Genomics. 2018; 19: 834

68. Koh A, Molinaro A, Stahlman M, Khan M T, Schmidt C, Manneras-Holm L, et al. Microbially Produced Imidazole Propionate Impairs Insulin Signaling through mTORC1. Cell. 2018; 175: 947-961 e17.

69. Moscat J, Diaz-Meco M T. p62 at the crossroads of autophagy, apoptosis, and cancer. Cell. 2009; 137: 1001-4

70. Koh A, Manneras-Holm L, Yunn N O, Nilsson P M, Ryu S H, Molinaro A, et al. Microbial Imidazole Propionate Affects Responses to Metformin through p38gamma-Dependent Inhibitory AMPK Phosphorylation. Cell Metab. 2020; 32: $643-653$ e4. 
71. Morales D R, Morris A D. Metformin in cancer treatment and prevention. Annu Rev Med. 2015; 66: 17-29.

72. Xiao-Jie L, Hui-Ying X, Zun-Ping K, Jin-Lian C, Li-Juan J. CRISPR-Cas9: a new and promising player in gene therapy. J Med Genet. 2015; 52: 289-96.

73. Vojta A, Dobrinic P, Tadic V, Bockor L, Korac P, Julg B, et al. Repurposing the CRISPR-Cas9 system for targeted DNA methylation. Nucleic Acids Res. 2016; 44: 5615-28.

74. Hilton I B, D'Ippolito A M, Vockley C M, Thakore P I, Crawford G E, Reddy T $\mathrm{E}$, et al. Epigenome editing by a CRISPR-Cas9-based acetyltransferase activates genes from promoters and enhancers. Nat Biotechnol. 2015; 33: 510-7.

75. Thakore P I, D'Ippolito A M, Song L, Safi A, Shivakumar N K, Kabadi A M, et al. Highly specific epigenome editing by CRISPR-Cas9 repressors for silencing of distal regulatory elements. Nat Methods. 2015; 12: 1143-9.

76. Doroshow D B, Bhalla S, Beasley M B, Sholl L M, Kerr K M, Gnjatic S, et al. PD-L1 as a biomarker of response to immune-checkpoint inhibitors. Nat Rev Clin Oncol. 2021.

77. Risco A, Martin-Serrano M A, Barber D F, Cuenda A. p38gamma and p38delta Are Involved in T Lymphocyte Development. Front Immunol. 2018; 9: 65. 